\title{
REVIEW \\ Study on Artificial Seeds of Plants
}

\author{
Shan Han ${ }^{1^{*}}$ Minran Yang ${ }^{2}$ Yanyi Chen ${ }^{2}$ \\ 1. Biological chemical, North China University of Science and Technology, Tangshan, Hebei, 063000, China \\ 2. Bioinformatics, North China University of Science and Technology, Tangshan, Hebei, 063000, China
}

\section{ARTICLE INFO}

Article history

Received: 15 July 2020

Accepted: 15 July 2020

Published Online: 30 September 2020

Keywords:

Embryoid body

Artificial seed

\begin{abstract}
The establishment and development of artificial seed technology is to quickly reproduce excellent varieties or hybrids, which can be applied to hybrid generation seeds produced by the three-two line method. For some varieties that are difficult to propagate with seeds or plant species with unstable genetic traits and poor fertility, artificial seed technology can also be used for mass reproduction. In particular, some new plants created through genetic engineering, such as somatic hybrids or transgenic plants, can be propagated or maintained by artificial seed technology. In addition, artificial seed technology can be used for the maintenance and rapid propagation of virus-free seedlings. Compared with ordinary test tube seedlings, artificial seeds have low cost, convenient transportation, and to a certain extent reduce vitrified seedlings.In particular, the production of artificial seeds does not occupy a large amount of soil. It can be produced all year round. Therefore, the research on artificial seeds has developed rapidly in the world.
\end{abstract}

been valued by many countries. After being buried in a certain capsule, the body has the function of seed and is directly used for sowing in the field.

The artificial seed technology in a broad sense includes the production and packaging of somatic embryos, storage of artificial seeds, artificial seed making equipment, etc., but in a narrow sense, artificial seed technology includes the production of somatic embryos and somatic cells Embryo wrapping (artificial endosperm and seed coat). The complete artificial seed includes three basic parts: somatic embryo, artificial endosperm, and artificial seed coat. Because artificial seeds are asexual in nature, they can propagate some plants that are not strong under natural conditions or have expensive seeds, fix

*Corresponding Author:

Han shan,

Biological chemical, North China University of Science and Technology, No. 21 Bohai Avenue, Caofeidian District, Tangshan, Hebei, 063000, China;

Email: china139331@126.com 
the heterosis, make Fl hybrids available for multiple generations, and enable excellent single plants to quickly reproduce. Clonal varieties, which greatly shortens the breeding period ${ }^{[5]}$.

Plant artificial seed is a bioengineering technology that is being created because of its great practical significance and economic value. At present, the United States, Japan, and France have carried out this research in succession. It has initially achieved success in plants such as carrots, celery, alfalfa, camellia, and tomatoes, but it is still in the laboratory stage and has not yet been applied to production practice.

\section{The Production Process of Artificial Seeds}

There are three major steps in making artificial seeds: (1) embryoid body culture; (2) wrap seed coat; (3) germination test.

Basic process: disinfection of the explant surface $\rightarrow$ induction of embryogenic callus $\rightarrow$ development of embryoid body $\rightarrow$ synchronization and screening of embryoid body $\rightarrow$ encapsulation of calcium alginate capsules by beading method $\rightarrow$ wrapping outer membrane $\rightarrow$ germination and seedling experiment.

To make artificial seeds, you must first have highquality embryoid bodies. Some plant tissue cultures are not easy to obtain embryoid bodies, but can also be replaced with adventitious buds, small bulbs, hairy roots, etc. But artificial seeds encapsulated with embryoid bodies are better. High-quality embryoid bodies can germinate and emerge in time after sowing, and the roots and shoots grow almost simultaneously. It has sufficient nutrients, has a certain resistance, and has strong vigor. The hypocotyl is not enlarged and there is no callus organization. The synchronization of development is good, and the size is basically the same after sorting. The germination and seedlings are neat, normal and non-deformed ${ }^{[6]}$.

\section{Embedding and Seed coat of Artificial Seeds}

The embedding methods of artificial seeds mainly include: liquid gel embedding method, dry wrapping method and hydrogel method.

The liquid gel embedding method is to directly suspend the embryoid bodies or small plants in the soil with a viscous fluid gel. Drew (1979) used this method to place a large number of carrot somatic embryos in a sugar-free nutrient matrix to obtain 3 plantlets. Baker (1985) then added sucrose to the liquid gum, and $4 \%$ of the embryos survived for 7 days. The drying method is a method of burying somatic embryos together with polyoxyethylene and other polymers. Although the method reported by Ktto et al. has a low plant formation rate, it demonstrates the effectiveness of drying and embedding somatic embryos. The hydrogel method refers to a method of encapsulating material formed by ion exchange or temperature mutation. Redenbaugh et al. (1987) used this method for the first time to obtain artificial seeds from somatic embryos of a single alfalfa plant, and the plant formation rate in vitro was $86 \%$. Later, this method was widely adopted by other artificial seed research groups ${ }^{[10]}$.

In order to solve the problem of human endosperm, people began to study the outer seed coat to add a protective layer. The ideal artificial seed coat should have: (1) Non-toxic and harmless to artificial seed embryos, biocompatible, and support artificial seed embryos; (2) Has a certain permeability and water retention, does not affect the storage and preservation of artificial seeds, Make the artificial seeds grow normally during germination; (3) Have a certain strength, can maintain the integrity of the capsule, which is convenient for the storage, transportation and sowing of artificial seeds; (4) Keep the nutritional ingredients and other auxiliary agents from leaking; (5) It can be degraded by some microorganisms (selective biodegradation), and the degradation products are not harmful to plants and the environment. ${ }^{2}$

RedenbaughThe others successfully used ElvexTM 4260 as the outer seed coat with a germination rate of $80 \%$.

Artificial seeds of Brassica oleracea were prepared with chitosan as the outer seed coat, and their germination rate was measured.But in the presence of bacteria, the germination rate is still not high. At present, most of the artificial seeds developed by the researchers use sterile agar germination test. Artificial (outer) seed coats are not necessary, so most artificial seeds do not have seed coats ${ }^{[9]}$.

\section{Conversion of Artificial Seeds under Soil Conditions}

Although the theory of somatic embryogenesis is important to restore a complete plant from a cell embryo under test-tube conditions, artificial seeds will eventually grow into a complete plant in a greenhouse or field. Many researchers have successfully obtained the same germination rate as the production conditions through experiments.

The germination of seeds in human soil, whether in a controlled box temperature environment or an uncontrolled greenhouse and field environment, requires the endurance of somatic embryos and the formation of its own roots, stems and leaves, also depends on the quality of the seedlings, high quality, Then the conversion of artificial seeds in the soil is high, and the subsequent 
development is also stable.

After the conversion of artificial seeds (growing seedlings), the main limiting factor for continuing to grow into a complete plant may be the availability of nutrients (salt and carbohydrates). To solve this problem, embryos must have their own nutrient reserves and external nutrition supplies. At the same time, these components are required for the entire growth period of the artificial seed rest system, so the nutritional structure library (seed coat, matrix) of the somatic embryo must have both endogenous (starch and protein) and exogenous (artificial endosperm $)^{[1]}$.

\section{Somatic Clone Variation of Artificial Seeds}

In plant cell tissue culture in vitro, tissue culture will produce clonal variation. Chromosome abnormalities and morphological variations also exist in the regenerated plants of wheat somatic cell culture. Vasil (1986) believed that the chromosome number and structure of the regenerated plants obtained by the somatic embryo route were stable. However, Hartman et al. (1987) found significant changes in the DNA of wheat embryonic cell lines. Bogini (1988) believed that the propagation of axillary buds could overcome somatic clone variation. The mutant was discovered during the germination of carrot artificial seeds. In addition, after the artificial seeds are dried, lowtemperature $\mathrm{ABA}$ treatment will increase the proportion of abnormal cotyledons. Japan Kirin Beer Company (1989) transplanted 20,000 seedlings of F1 generation of artificial seeds, celery and lettuce into the field, observed the offspring, and found no changes in the production and quality of artificial seed germination seedlings.

Artificial seed germination seedling variation is strived to avoid in the application of artificial seeds, but did not overcome the changes of effective means. However, if the mutation trait is not important to the crop, or if the mutation frequency is lower than the allowable value that affects yield, this artificial seed is still valuable.

For most crops, artificial seeds are not as good as natural seeds, but with the advancement of artificial seed technology, in some crops, it is possible to replace natural seeds with artificial seeds ${ }^{[8]}$.

\section{The Significance and Prospect of Artificial Seeds}

Seeds are the basis for plant seed generation and human reproduction. Artificial seeds not only can be stored, transported, sown, germinated and grown into normal plants like natural seeds, but also have many unique advantages.

(1) Plants that are not strong or have expensive seeds can be propagated under natural conditions. (2) Fixed heterosis. (3) Fast and efficient breeding method. (4) The research results of genetic engineering can be transformed into productivity as soon as possible. (5) The growth and development and stress resistance of plants can be controlled artificially. (6) Low cost, convenient storage and transportation ${ }^{[7]}$.

Artificial seeds have shown attractive prospects to humans since their birth, but there are still many problems (including theoretical, technical, and commercial) in large-scale application in production that need to be solved urgently. Artificial seeds must really enter the commercial market and allow nature Seed competition, the most important thing is to reduce production costs, because the cost of artificial seeds of most crops is still much higher than natural seeds, but one thing is clear, that is, artificial seeds and test tube seedlings use less medium, Small size, fast reproduction, fast germination and seedling generation, convenient transportation and storage, the prospect of the development and utilization of artificial seeds is attractive. It is foreseeable that this biological high-tech will play a huge role in crop genetic breeding, improved breeding and cultivation ${ }^{[3]}$.

\section{References}

[1] Hongbo Shao, Liye Chu. Advances in Plant Artificial Seed Research. Guangxi Plant, 1990: 168-74.

[2] Shaohuan Ding, Mingsheng Zhang, Mengna Shi. Advances in Research on Plant Artificial Seed Technology. Seed, 2011, 30: 60-66.

[3] Lanfang Du. Artificial Plant Seeds. Shanghai Agricultural Science and Technology, 1995: 48-50.

[4] Zhongchen Guo, Jiyi Jin. A Preliminary Report on the Research of Plant Artificial Seeds. Botany Bulletin, 1988, 5.

[5] Xincen Liu, Guosheng Fan. Advances in Research and Application of Plant Artificial Seeds. Green Technology, 2010, 39-41.

[6] Yu Luo. Artificial Plant Seeds. Yunnan Agricultural Science and Technology, 2000, 42-45.

[7] Jialin Wang. Summary of Plant Tissue Culture and Its Application Research. Heilongjiang Agricultural Science, 2006, 86-89.

[8] Xiaohei Xing, Daochuan Yin, Qiucheng Yu. Advances in Plant Artificial Seed Research. Biotechnology, 1993, 1-5.

[9] Shuiying Ye, Tao Wan. Prospects for Production and Application of Artificial Seeds. Beijing Agriculture, 2012, 186-87.

[10] Ming Zhang, Huarong Huang, Xiaoyong Wei. Advances in Plant Artificial Seed Research. Bulletin of Botany, 2000, 407-12. 